\title{
Broadly neutralizing antibodies with few somatic mutations and hepatitis C virus clearance
}

Justin R. Bailey, ${ }^{1}$ Andrew I. Flyak, ${ }^{2}$ Valerie J. Cohen, ${ }^{1}$ Hui Li, ${ }^{3}$ Lisa N. Wasilewski, ${ }^{1}$ Anna E. Snider, ${ }^{1}$ Shuyi Wang, ${ }^{3}$ Gerald H. Learn, ${ }^{3}$ Nurgun Kose, ${ }^{4}$ Leah Loerinc, ${ }^{4}$ Rebecca Lampley, ${ }^{4}$ Andrea L. Cox, ${ }^{1,5}$ Jennifer M. Pfaff, ${ }^{6}$ Benjamin J. Doranz, ${ }^{6}$ George M. Shaw, ${ }^{3,7}$ Stuart C. Ray, ${ }^{1,5}$ and James E. Crowe Jr. ${ }^{2,4,8}$

'Department of Medicine, Johns Hopkins University, Baltimore, Maryland, USA. ²Department of Pathology, Microbiology, and Immunology, Vanderbilt University Medical Center, Vanderbilt University, Nashville, Tennessee, USA. ${ }^{3}$ Department of Medicine, University of Pennsylvania, Philadelphia, Pennsylvania, USA. ${ }^{4}$ Vanderbilt Vaccine Center, Vanderbilt University, Nashville, Tennessee, USA. ${ }^{5}$ Department of Oncology, Johns Hopkins University, Baltimore, Maryland, USA. ${ }^{6}$ Integral Molecular Inc., Philadelphia, Pennsylvania, USA. 'Department of Microbiology, University of Pennsylvania, Philadelphia, Pennsylvania, USA. ${ }^{8}$ Department of Pediatrics, Vanderbilt University Medical Center, Vanderbilt University, Nashville, Tennessee, USA.

Here, we report the isolation of broadly neutralizing mAbs (bNAbs) from persons with broadly neutralizing serum who spontaneously cleared hepatitis $C$ virus (HCV) infection. We found that bNAbs from two donors bound the same epitope and were encoded by the same germline heavy chain variable gene segment. Remarkably, these bNAbs were encoded by antibody variable genes with sparse somatic mutations. For one of the most potent bNAbs, these somatic mutations were critical for antibody neutralizing breadth and for binding to autologous envelope variants circulating late in infection. However, somatic mutations were not necessary for binding of the bNAb unmutated ancestor to envelope proteins of early autologous transmitted/founder viruses. This study identifies a public B cell clonotype favoring early recognition of a conserved HCV epitope, proving that anti-HCV bNAbs can achieve substantial neutralizing breadth with relatively few somatic mutations, and identifies HCV envelope variants that favored selection and maturation of an anti-HCV bNAb in vivo. These data provide insight into the molecular mechanisms of immune-mediated clearance of $\mathrm{HCV}$ infection and present a roadmap to guide development of a vaccine capable of stimulating anti-HCV bNAbs with a physiologic number of somatic mutations characteristic of vaccine responses.

Authorship note: J.R. Bailey and A.I. Flyak contributed equally to this work.

Conflict of interest: The authors have declared that no conflict of interest exists.

Submitted: January 17, 2017

Accepted: March 21, 2017

Published: May 4, 2017

Reference information: JCI Insight. 2017;2(9):e92872. https:// doi.org/10.1172/jci.insight.92872

\section{Introduction}

Hepatitis C virus (HCV) infects approximately 185 million people worldwide and is a major cause of liver failure and hepatocellular carcinoma (1). With the recent development of potent, oral interferon-free therapies, treatment of HCV infection has improved significantly. However, HCV eradication is unlikely to be achieved with treatment alone. Identification of those with HCV infection is challenging. Therapies are too costly for countries with the highest incidence. Reinfection can occur following treatment, and transmission of drug-resistant $\mathrm{HCV}$ is possible (2). The rate of acute HCV infection increased in most US states between 2010 and 2014, following an ongoing epidemic in opioid/heroin use (3-5). This rising epidemic of acute HCV infection in the US gives new urgency to prophylactic vaccine development efforts.

Broadly neutralizing human mAbs (bNAbs) capable of neutralizing diverse HCV strains have been isolated from HCV-infected individuals, proving that antibodies can target relatively conserved regions of the two HCV envelope glycoproteins (E1 and E2), despite the enormous genetic diversity of HCV (6-17). Infusion of bNAbs is protective against infection in animal models of $\operatorname{HCV}(17,18)$, and a recent study also showed that bNAbs could abrogate established HCV infection in a humanized transgenic mouse model (19). Given the efficacy of these bNAbs in blocking HCV infection, the molecular and genetic features of bNAbs and their epitopes may serve as a useful guide for rational HCV vaccine design. 
Studies of the evolution of HIV-specific bNAbs have enabled an entire field of germline-targeted vaccine designs and stabilization of envelope antigens (20-22). However, studies of the natural evolution of HIV bNAbs still may not be the optimal method for fully understanding the fundamental principles of breadth and potency for bNAbs, because HIV-infected individuals do not clear their infections. In contrast, approximately $30 \%$ of individuals who become infected with HCV spontaneously clear the infection (23), even though the viral diversity in HCV-infected individuals is comparable to or exceeds that of the diversity of HIV isolates in HIV-infected subjects (24-27). Spontaneous clearance of HCV has been associated with effective innate and T cell responses, but we and others have shown that spontaneous clearance is associated with early appearance of broadly neutralizing antibodies against $\mathrm{HCV}$ in serum $(28,29)$. mAbs from individuals with broadly neutralizing serum and clearance of HCV have not been isolated to date, so it is not known whether these mAbs have unique features relative to the $\mathrm{mAbs}$ previously isolated from individuals with persistent HCV infection. It is of interest to define the molecular basis for recognition and neutralization of an entire quasispecies of an antigenically diverse virus like $\mathrm{HCV}$, with subsequent immune-mediated clearance.

To study this mechanism, we have followed prospectively a cohort of subjects from a time point prior to infection through the time of their spontaneous clearance of HCV. In this study, we isolated a panel of bNAbs from two of these subjects who spontaneously cleared HCV infection. We characterized the neutralizing breadth of these bNAbs, mapped the targeted epitopes, identified a germline heavy chain variable gene segment that was used by multiple bNAbs, and identified somatic mutations in one bNAb that were critical for breadth of recognition of heterologous envelope variants. We also defined the longitudinal evolution of the virus in the donor of this $\mathrm{bNAb}$, allowing us to show that the bNAb unmutated ancestor could bind envelope proteins of early autologous transmitted/founder (T/F) viruses and the mature bNAb could bind variants circulating immediately prior to viral clearance. Defining these determinants of bNAb breadth and the critical antigenic determinants favoring $b N A b$ induction provides insight into molecular mechanisms of immune-mediated clearance of $\mathrm{HCV}$ infection and informs rational vaccine design.

\section{Results}

Identification of subjects who spontaneously cleared HCV infection and possess broadly neutralizing circulating antibodies. We developed a diverse panel of 19 genotype $1 \mathrm{a}$ and $1 \mathrm{~b} \mathrm{HCV}$ pseudoparticles (HCVpp) that allowed us to screen donor plasma for HCV neutralizing breadth $(28,30)$. This panel comprises $94 \%$ of the amino acid polymorphisms present at greater than $5 \%$ frequency in a reference panel of 643 genotype $1 \mathrm{HCV}$ isolates from GenBank. In a previous study, we used this panel to screen plasma samples isolated prior to viral clearance from 21 subjects who spontaneously cleared HCV infection or 42 duration-of-infection-matched plasma samples from subjects with persistent infection (28). We identified two subjects who spontaneously cleared HCV and who possessed exceptionally broad plasma neutralizing antibody activity. Subject 117 was HCV plasma antibody negative and HCV RNA negative on study enrollment before becoming infected with genotype 1a HCV. Subject 110 was initially viremic, also with genotype 1a virus, but was HCV serum antibody negative, indicating recent infection. Both subjects exhibited typical high viral loads during acute infection, followed by a subsequent viral load decline and eventual clearance of the infection (Figure 1A). Subject 117 was infected for about 1 year total prior to clearance. Subject 110 had a more unusual disease course, with high viral loads for about 1 year, followed by a drop in viral load, years of extremely low-level intermittent viremia, and eventual viral clearance. Repeated sequencing of viral structural genes throughout infection did not reveal any large genetic shifts in either subject that would suggest superinfection or reinfection with a new viral strain. After clearance, each subject was followed for more than 5 years with frequent viral load testing, with no recurrence of viremia. Plasma of subject 117 was tested for neutralizing antibody breadth at day 388 after infection, the last time point prior to viral clearance. Plasma of subject 110 was tested at day 401 after infection, the time point immediately preceding a decline of $10^{7} \mathrm{IU} / \mathrm{ml}$ in viral load. Using a threshold of at least $50 \%$ neutralization by a 1:100 dilution of plasma, the plasma of subject 117 neutralized 14 of $19 \mathrm{HCVpp}$ variants (74\%) and the plasma of subject 110 neutralized 17 of 19 variants (89\%) (Figure 1B). For comparison, the median percentage of neutralization by time-matched plasma samples from 42 subjects with persistent infection did not exceed $50 \%$ for any of the 19 HCVpp tested.

Isolation of neutralizing $m A$ bs and measurement of neutralizing breadth. We isolated peripheral blood mononuclear cells (PBMCs) from subjects 110 and 117 after HCV clearance. Using previously established methods (see Methods), we transformed B cells in PBMC samples with EBV and screened the transformed B 

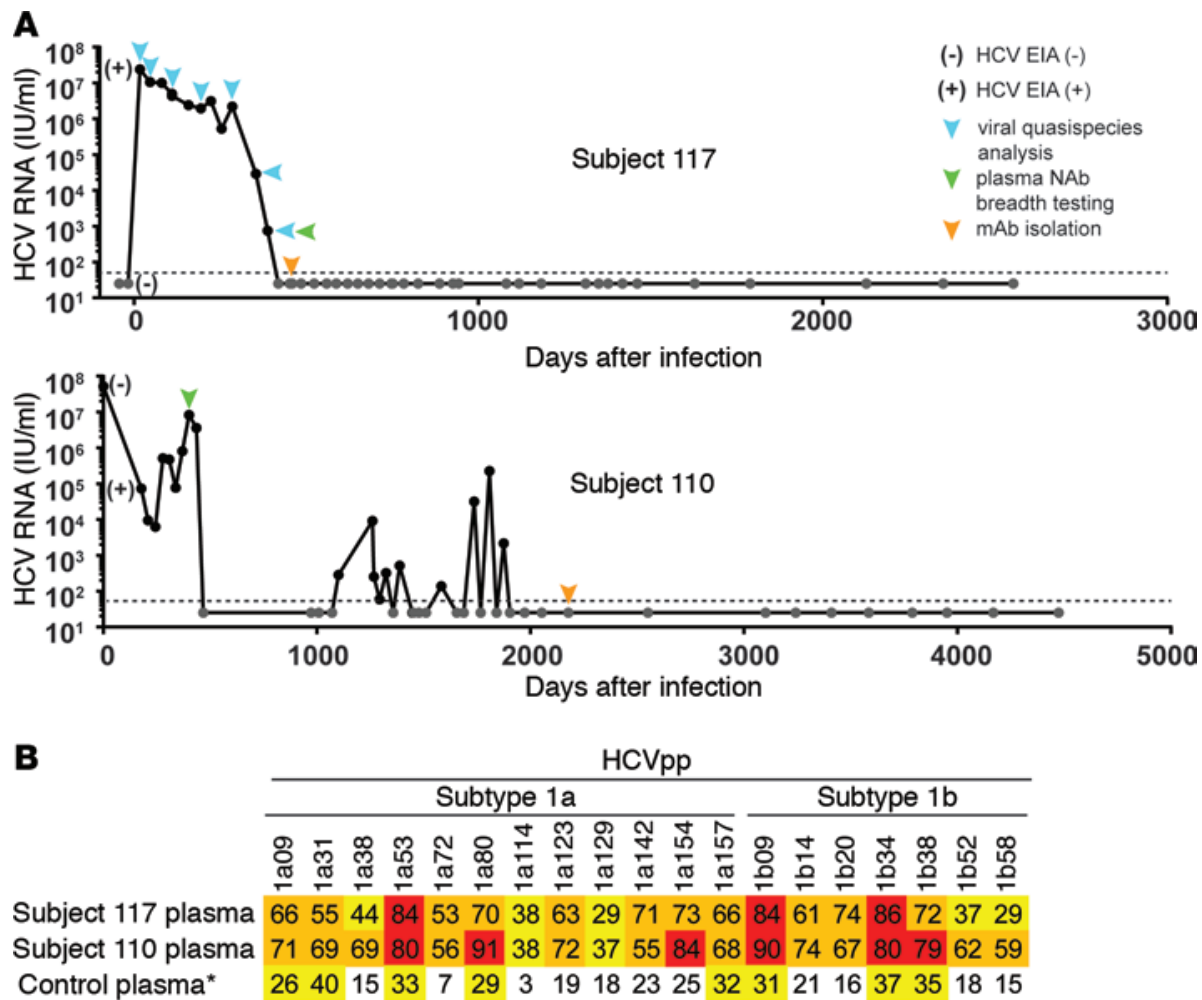

\begin{tabular}{|l|l|l|l|l|}
\hline \multirow{2}{*}{$\%$ Neutralization } & & & & \\
\cline { 2 - 5 } & $>75$ & $50-75$ & $25-50$ & $<25$ \\
\hline
\end{tabular}

Figure 1. Identification of subjects who spontaneously cleared HCV and possess broadly neutralizing antibodies in plasma. (A) HCV viral loads of two subjects who spontaneously cleared HCV infection sampled periodically from the time of initial infection through viral clearance. The dashed line indicates limit of detection (LOD) of the viral load assay, which is $50 \mathrm{IU} / \mathrm{ml}$. Values below the LOD are set at $25 \mathrm{IU} / \mathrm{ml}$ and marked with gray circles. Plasma samples that were HCV antibody negative or positive by commercial antibody-binding assay (EIA) are indicated by - and +, respectively. The green triangles represent plasma samples tested for neutralizing breadth against the panel of 19 genotype 1 HCV pseudoparticle (HCVpp). The yellow triangles represent time points from which anti-HCV mAbs were isolated. The blue triangles represent plasma samples from which the viral quasispecies was sequenced by single-genome amplification. (B) Neutralizing breadth of plasma from the two subjects tested against a diverse panel of genotype 1a or $1 \mathrm{~b}$ HCVpp. Values shown are the percentage of neutralization achieved by a 1:100 dilution of plasma, tested in duplicate. ${ }^{*}$ For comparison, the median neutralization of each HCVpp from 42 subjects with persistent HCV infection, matched with subjects who cleared for duration of infection (Control plasma), is also shown.

lymphoblastoid cell line culture supernatants for neutralizing activity against one autologous HCV variant and a second heterologous variant. Cells from wells with neutralizing activity against either variant were fused with myeloma cells to produce hybridomas, which then were cloned biologically using limiting dilution and flow cytometric sorting and repeated screening of culture supernatants for HCV E1E2 proteinbinding activity. We isolated 15 anti-E1E2 mAbs from the two subjects. Purified mAbs were tested for intragenotypic neutralizing breadth against the panel of 19 genotype $1 \mathrm{HCVpp}$, using a threshold of $\geq 50 \%$ reduction as the definition of neutralization. The most broadly neutralizing mAbs are shown in Figure 2, with the remaining mAbs shown in Supplemental Figure 1 (supplemental material available online with this article; https://doi.org/10.1172/jci.insight.92872DS1). For comparison, two previously described bNAbs, AR4A and AR3C $(9,17)$, were tested against the same viral panel. The most broadly neutralizing mAbs isolated from subject 117, designated HEPC 3 and HEPC84, neutralized 16 of 19 (84\%) or 11 of 19 (58\%) of variants, respectively. HEPC43 and HEPC74, the most broadly neutralizing mAbs isolated from subject 110, each neutralized 17 of $19(90 \%)$ variants. For comparison, AR3C and AR4A neutralized 16 of $19(84 \%)$ and 12 of $19(63 \%)$ variants, respectively. Next, we measured cross-genotypic neutralizing titers of mAbs HEPC3, HEPC43, HEPC74, or HEPC84 against a standard panel of genotype 1-6 replication competent cell culture viruses (HCVcc) (Figure 3). HEPC3, HEPC43, and HEPC74 each neutralized variants from 5 of 6 genotypes, and HEPC 84 neutralized variants from 4 of 6 genotypes. None of the mAbs showed 


\begin{tabular}{|c|c|c|c|c|c|c|c|c|c|c|c|c|c|c|c|c|c|c|c|c|}
\hline & \multicolumn{19}{|c|}{ HCVpp } \\
\hline & & \multicolumn{12}{|c|}{ Subtype 1a } & \multicolumn{7}{|c|}{ Subtype $1 \mathrm{~b}$} \\
\hline & & 욤 & $\underset{\widetilde{\Phi}}{\sigma}$ & 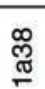 & 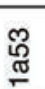 & 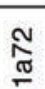 & ષ્ષ & $\stackrel{0}{\frac{\sigma}{\sigma}}$ & $\frac{\text { ্ָ }}{\text { త్ }}$ & $\frac{\mathbb{\Phi}}{\mathbb{\sigma}}$ & $\frac{\text { I }}{\frac{\pi}{\sigma}}$ & $\frac{\mathbb{\sigma}}{\sigma}$ & $\frac{\sqrt{n}}{\sigma}$ & $\begin{array}{l}\text { 옹 } \\
\stackrel{0}{\circ}\end{array}$ & $\stackrel{+}{\stackrel{0}{0}}$ & ָㅗㄹ & 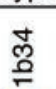 & 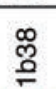 & $\begin{array}{l}\text { N్ } \\
\stackrel{0}{\rightleftharpoons}\end{array}$ & $\begin{array}{l}\stackrel{\infty}{p} \\
\stackrel{p}{\rho}\end{array}$ \\
\hline \multirow{7}{*}{ } & AR4A & 31 & 70 & 11 & 85 & 60 & 73 & 30 & 88 & 29 & 79 & 95 & 57 & 93 & 68 & -2 & 92 & 70 & 38 & 22 \\
\hline & AR $3 C$ & 63 & 87 & 61 & 91 & 71 & 57 & 21 & 61 & 49 & 68 & 78 & 79 & 97 & 82 & 53 & 81 & 88 & 71 & 17 \\
\hline & HEPC3 & 79 & 85 & 76 & 91 & 83 & 64 & 27 & 72 & 49 & 78 & 82 & 54 & 96 & 81 & 87 & 84 & 93 & 77 & 12 \\
\hline & HEPC 43 & 83 & 82 & 84 & 94 & 83 & 67 & 5.9 & 72 & 62 & 69 & 56 & 74 & 97 & 86 & 67 & 89 & 92 & 85 & 2.5 \\
\hline & HEPC74 & 87 & 77 & 82 & 99 & 82 & 75 & 8 & 82 & 75 & 83 & 94 & 89 & 99 & 93 & 96 & 94 & 97 & 88 & 48 \\
\hline & HEPC 84 & 57 & 79 & 39 & 68 & 59 & 33 & 61 & 69 & 12 & 64 & 71 & 43 & 79 & 7.7 & 55 & 65 & 18 & -11 & -27 \\
\hline & HEPC98 & 1.6 & 16 & 80 & 93 & 11 & 96 & 91 & -13 & 1.5 & 35 & 100 & 98 & -5 & 5.4 & -3 & 3.6 & 0.1 & -1 & -20 \\
\hline
\end{tabular}

$\%$ Neutralization

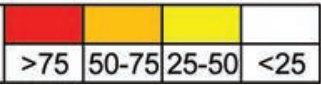

Figure 2. Intragenotypic neutralizing breadth of anti-HCV mAbs isolated from two subjects who spontaneously cleared HCV infection. Neutralizing breadth of mAbs against a diverse panel of genotype 1a or $1 \mathrm{~b}$ HCV pseudoparticles (HCVpp). Neutralization patterns for the 5 most broadly neutralizing mAbs are shown; data for the remaining 10 mAbs are shown in Supplemental Figure 1. mAbs marked with blue were isolated from subject 117 and mAbs marked with green were isolated from subject 110 . Values shown are the percentage of neutralization achieved by $50 \mu \mathrm{g} / \mathrm{ml} \mathrm{mAb}$. Values are means of two independent experiments, each performed in duplicate. For reference, previously described bNAbs AR4A and AR3C were tested in parallel against the same HCVpp panel.

potent neutralizing activity against the genotype 3 isolate (S52), which, interestingly, is also true of many other previously described broadly neutralizing anti-HCV human mAbs (17). HEPC3, HEPC43, HEPC74, and HEPC 84 each bound native E1E2 protein in an ELISA but did not bind to denatured protein, indicating that they bind conformational epitopes on E1E2 (Supplemental Figure 2).

Epitope mapping. mAb-binding epitopes were mapped using comprehensive alanine-scanning mutagenesis across E1E2. Six mAbs displayed adequate binding to the target variant, strain H77 E1E2, to allow mapping by this method (Figure 4 and Supplemental Figure 3). HEPC3, HEPC43, and HEPC74 displayed overlapping epitopes with multiple shared binding residues. Surprisingly, the binding epitope of these mAbs also overlapped with the epitope of the previously described $\mathrm{bNAb}$, AR3C, which was determined by cocrystallization of AR3C Fab with HCV E2 core (31). Two of the binding residues shared by HEPC3, HEPC43, and HEPC74 (aa 530 and 535) also have been shown to be critical for interaction with the HCV receptor CD81, suggesting that escape mutations at these positions might adversely affect viral fitness (32). We further mapped the binding epitopes of these mAbs by measuring competition for E1E2 binding among the mAbs or between the mAbs and a panel of previously characterized reference bNAbs. The six most broadly neutralizing mAbs are shown in Figure 4, B and C, with all mAbs shown in Supplemental Figure 4. HEPC3, HEPC43, and HEPC74 showed reciprocal competition for binding with each other, and HEPC82 reduced binding of HEPC84 and HEPC98. As expected from the alanine-scanning maps, reference $\mathrm{mAb} A \mathrm{AR} 3 \mathrm{C}$ reduced binding of HEPC3, HEPC43, and HEPC74, suggesting that the mAbs compete for overlapping binding sites. Reference mAb AR4A, which binds only to complexed E1 and E2, reduced binding of the mAbs HEPC82 and HEPC84. Reference mAb HC33.8 (8), which targets an epitope spanning amino acids 408-423, competed with $\mathrm{mAb}$ HEPC98, which has binding residues at amino acids 402, 405, and 408 .

In addition, we characterized relationships between bNAbs using a neutralization profiling method that has been used extensively for characterization of anti-HIV mAbs (33). We recently adapted this method to allow grouping of HCV mAbs into functionally related clusters based upon relative neutralizing capacity across a genetically diverse panel of HCV variants (30). In this analysis, we compared neutralization values of the previously uncharacterized mAbs across the panel of $19 \mathrm{HCVpp}$ to each other and to a large panel of previously described reference bNAbs (30) using Spearman correlations (Figure 4D and Supplemental Figure 5). HEPC3, HEPC43, and HEPC74 clustered most closely with each other, and, of the 18 reference bNAbs tested, they clustered most closely with mAb AR3C, in agreement with the alanine-scanning and competition-binding data. Consistent with the competition-binding results, HEPC84 clustered most closely with mAb AR4A and HEPC98 clustered near mAbs HC33.4 and HC33.8. Taken together, these three mapping studies show remarkable similarity between the binding 

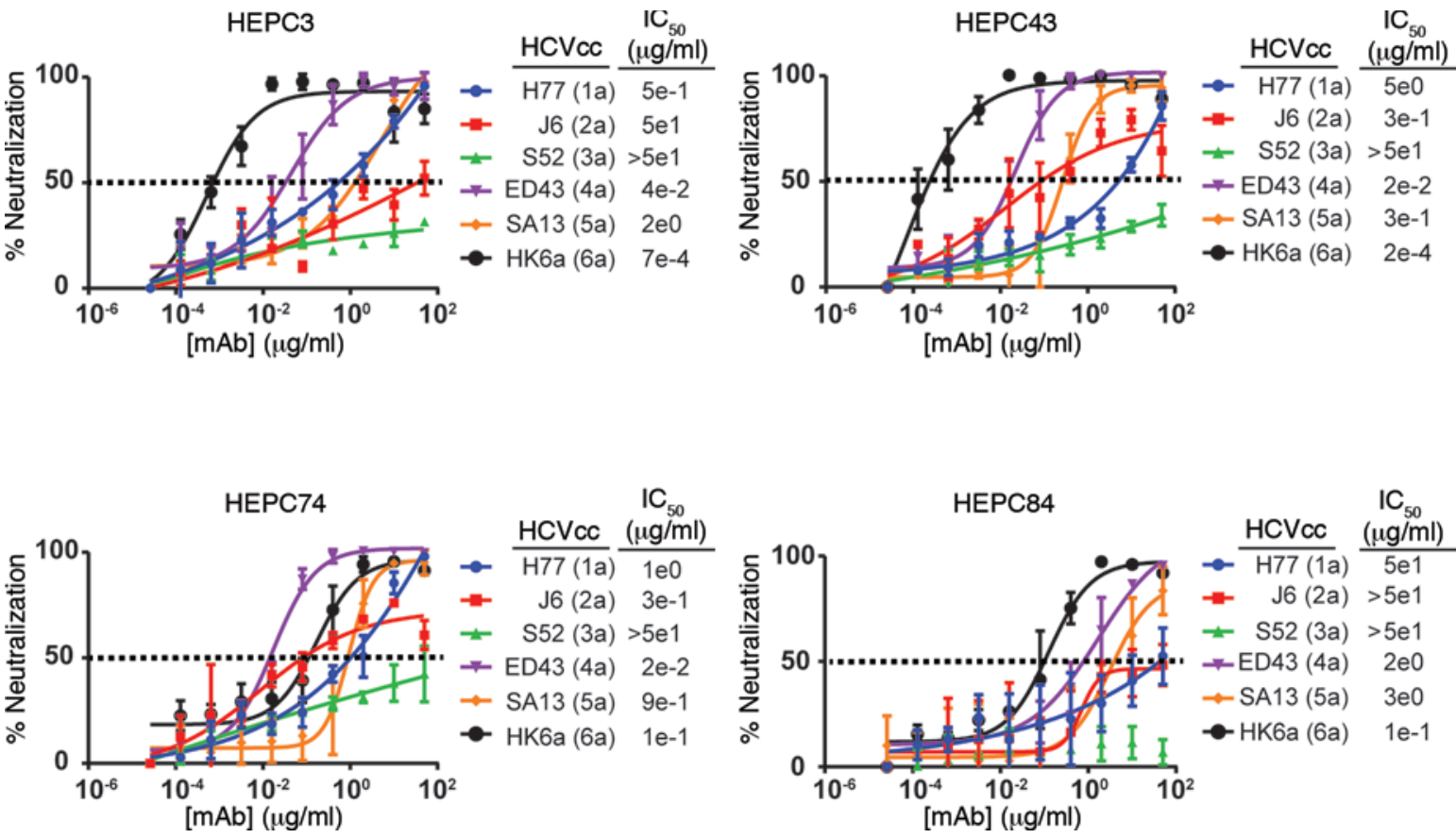

Figure 3. Cross-genotypic neutralizing breadth of anti-HCV mAbs. Neutralizing breadth of mAbs against a panel of genotype 1-6 replication competent hepatitis C viruses (HCVcc). The 4 mAbs with greatest neutralizing breadth in Figure 2 were tested. The name of each HCVcc strain is indicated, with the viral subtype in parenthesis. Values shown are the means of two independent experiments, each performed in triplicate, and error bars represent standard deviations between experiments. The half-maximal inhibitory concentration $\left(\mathrm{IC}_{50}\right)$ of each $\mathrm{mAb} / \mathrm{HCV} c \mathrm{c}$ combination is shown. Curves with neutralization exceeding $50 \%$ at only the highest mAb concentration ( 50 $\mu \mathrm{g} / \mathrm{ml}$ ) were assigned an $\mathrm{IC}_{50}$ of $50 \mu \mathrm{g} / \mathrm{ml}$.

epitopes of mAbs HEPC3, HEPC43, and HEPC74, which were isolated from two different subjects who spontaneously cleared HCV infection, and AR3C, a previously described bNAb isolated from a patient with persistent $\mathrm{HCV}$ infection (17). Based upon competition-binding data and neutralization profiling, HEPC84 and the reference mAb AR4A also appear to bind to overlapping epitopes, as do HEPC98 and the reference $\mathrm{mAb} \mathrm{HC} 33.8$.

Somatic mutations and antibody breadth. We sequenced the heavy and light chain variable gene sequences of each of the mAbs. Surprisingly, despite developing in two different individuals, HEPC3, HEPC43, and HEPC74 are each encoded by the same antibody heavy chain variable gene segment, $\mathrm{V}_{\mathrm{H}} 1-69$ (Table 1). Of note, $\mathrm{mAb} A R 3 \mathrm{C}$, which was isolated from a subject with chronic $\mathrm{HCV}$ infection, also uses $\mathrm{V}_{\mathrm{H}} 1-69$ (17), as do some other anti-HCV bNAbs $(12,34)$. Together with the epitope mapping data, these results suggest that $\mathrm{V}_{\mathrm{H}} 1-69$ encodes antibodies with capacity to form a public $\mathrm{B}$ cell clonotype that favors binding to an immunodominant broadly neutralizing HEPC3/AR3C epitope. Remarkably, these bNAbs were encoded by antibody genes with sparse somatic mutations. HEPC 3 has $95 \%$ identity to its germline heavy chain $\mathrm{V}_{\mathrm{H}}$ gene sequence, while HEPC 43 has $95 \%$ and HEPC 74 has $92 \%$ identity to its germline heavy chain $\mathrm{V}_{\mathrm{H}}$ gene sequence. The light chains encoding these $m A b s$ have $96 \%-98 \%$ identity to their germline $V_{L}$ genes. By comparison, $m A b$ AR3C, isolated previously from a subject with chronic $\mathrm{HCV}$ infection, has only $86 \%$ identity to its $\mathrm{V}_{\mathrm{H}}$ gene (17), meaning that the bNAbs isolated from subjects who spontaneously cleared infection have substantially fewer $\mathrm{V}_{\mathrm{H}}$ gene somatic mutations than AR3C. In addition, these $\mathrm{mAbs}$ have substantially fewer somatic mutations than most previously described anti-HIV bNAbs, including VRC01 (22), which has only $68 \%$ identity to its germline heavy chain $\mathrm{V}_{\mathrm{H}}$ gene sequence.

To identify somatic mutations critical for bNAb breadth, we mutated all 13 somatic mutations in the HEPC 3 heavy chain, all 7 somatic mutations in the light chain, or both, to match the inferred germline sequence, generating HEPC3 reverted unmutated ancestor (RUA) variants designated H-RUA, L-RUA, and H,L-RUA, respectively. We measured the ability of mature HEPC3, H-RUA, L-RUA, and H,L-RUA antibodies to neutralize a diverse panel of genotype $1 \mathrm{E} 1 \mathrm{E} 2$ variants (Figure $5 \mathrm{~A}$ ). As in prior tests, mature HEPC3 neutralized the majority of variants in the panel. HEPC3 L-RUA showed nearly identical neutral- 
A

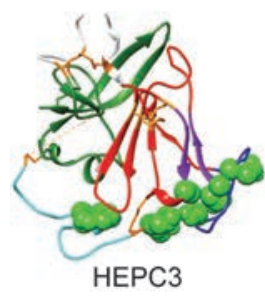

1

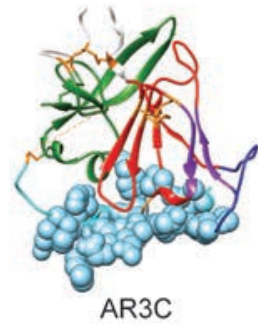

AR3C

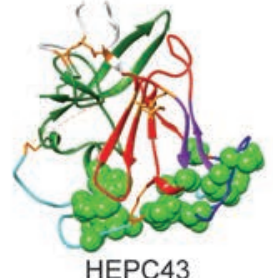

HEPC43

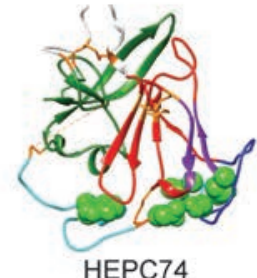

HEPC74
B

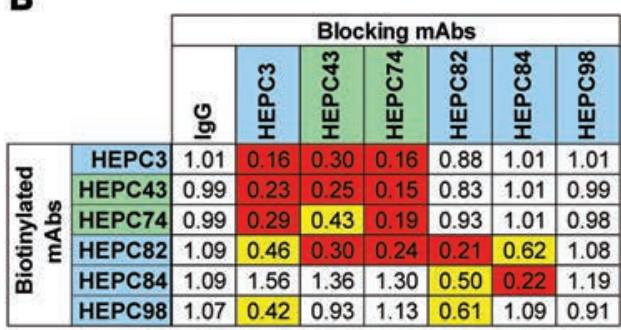

C
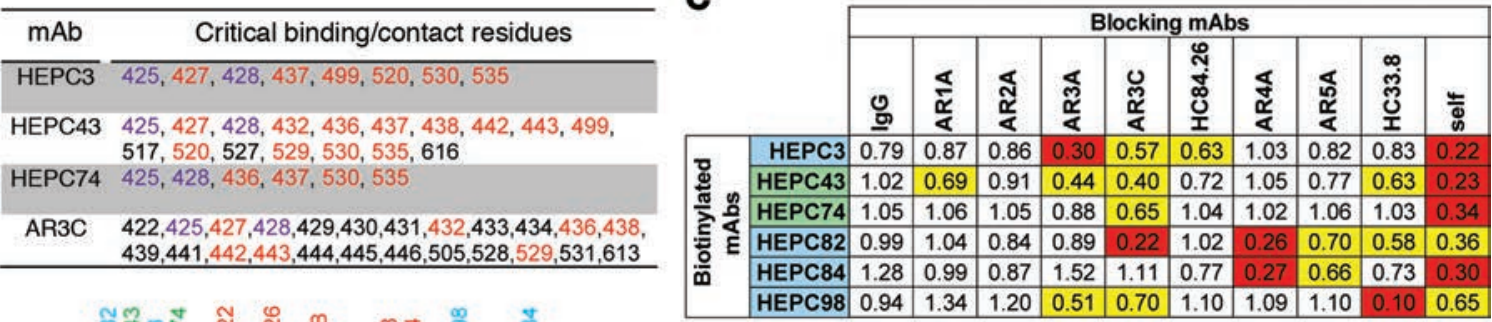

D

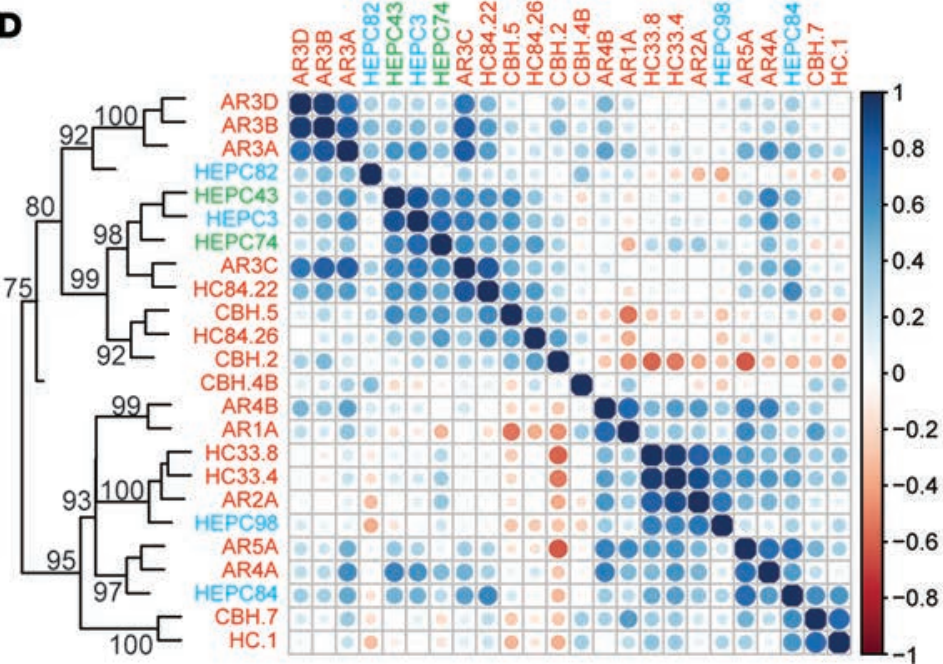

Figure 4. Epitope mapping of anti-HCV bNAbs. (A) Critical binding residues for bNAbs based on relative binding to alanine-scanning mutants spanning the full H77 E1E2 sequence. Binding residues are marked with green spheres superimposed on the H77 E2 core structure (31). For reference, contact residues for mAb AR3C, identified by Kong et al., are indicated with blue spheres. Additional mAbs are shown in Supplemental Figure 3. In the table, critical binding or contact residues shared by at least two mAbs are highlighted in red, and those shared by all $4 \mathrm{mAbs}$ in purple. (B and C) Competition binding between mAbs. The 6 most broadly neutralizing mAbs from s117 (blue) and s110 (green) are shown, with additional mAbs shown in Supplemental Figure 4. Relative binding of $2 \mu \mathrm{g} / \mathrm{ml}$ of the biotinylated mAbs to strain $1 \mathrm{a} 53 \mathrm{E} 1 \mathrm{E} 2$ in the presence or absence of blocking $\mathrm{mAbs}$ at a concentration of $20 \mu \mathrm{g} / \mathrm{ml}$. Combinations resulting in relative binding $<0.7$ or $<0.35$ are marked in yellow or red, respectively. (B) Competition binding between newly identified $\mathrm{mAbs}$ and each other. Values represent the average of two independent experiments performed in duplicate. (C) Competition binding between newly identified $m A$ bs and reference bNAbs. Values represent the average of replicates from one experiment, except for AR3C, which was tested in duplicate in two independent experiments. (D) Clustering of the 6 most broadly neutralizing mAbs (blue or green) with reference bNAbs (red) based upon neutralization profiling. For each mAb, neutralization of each of $19 \mathrm{HCV}$ pseudoparticles was measured, generating a neutralization profile, and pairwise Spearman correlations were measured between these neutralization profiles. Circles at each intersection were scaled by the magnitude of the correlation between the indicated mAbs. Hierarchical clustering analysis using these pairwise correlations is depicted as a tree. Numbers at tree nodes are approximately unbiased (AU) test values (49), indicating strength of support for a particular cluster.

ization results, indicating that somatic mutations in the light chain are not critical for neutralizing breadth. Neutralizing breadth of both HEPC 3 H-RUA and H,L-RUA was greatly attenuated, indicating that somatic mutations in the heavy chain are critical for HEPC3 neutralizing breadth. Consistent with the neutralization data, mature HEPC3 bound all heterologous E1E2 variant proteins in an ELISA (Figure 5B). There was little reduction in binding of HEPC 3 L-RUA relative to mature HEPC3. Binding of HEPC3 H-RUA and H,L-RUA to all E1E2 proteins was significantly reduced, confirming that somatic mutations in heavy chain CDR1, CDR2, and/or CDR3 are critical for binding affinity and breadth of HEPC3.

To identify individual somatic mutations that are important for breadth of HEPC3, we also performed site-directed mutagenesis to revert each heavy chain somatic mutation individually to the germline-encoded 
Table 1. Heavy and light chain variable gene usage

\begin{tabular}{|c|c|c|c|c|c|}
\hline Donor & $\mathbf{m A b}$ & $V_{H}$ gene & nt identity (\%) & $V_{L}$ gene & nt identity $(\%)^{A}$ \\
\hline \multirow[t]{11}{*}{117} & НЕРСЗ & $1-69$ & 95 & Vк1-39 & 96 \\
\hline & HEPC80 & $3-49$ & 96 & Vк3-11 & 98 \\
\hline & HEPC82 & $4-31$ & 98 & $V \lambda 6-57$ & 97 \\
\hline & HEPC84 & $3-49$ & 94 & Vк1-39 & 94 \\
\hline & HEPC85 & $3-30$ & 90 & $V \kappa 2-29$ & 95 \\
\hline & HEPC87 & $4-31$ & 93 & $V \lambda 6-57$ & NA \\
\hline & HEPC90 & $3-33$ & 94 & $V \kappa 3-15$ & 98 \\
\hline & HEPC91 & $3-30$ & 86 & $V_{\kappa} 2-29$ & 92 \\
\hline & HEPC96 & $4-30$ & 95 & $V_{\kappa} 3-20$ & 98 \\
\hline & HEPC97 & $4-34$ & 92 & $V \lambda 3-9$ & NA \\
\hline & HEPC98 & $1-69$ & 92 & $V \lambda 3-25$ & 91 \\
\hline \multirow[t]{4}{*}{110} & HEPC43 & $1-69$ & 95 & $V \lambda 7-46$ & 98 \\
\hline & HEPC46 & $1-18$ & 97 & $V \lambda 1-47$ & 98 \\
\hline & HEPC50 & $1-58$ & 98 & $V_{\kappa} 2-28$ & 99 \\
\hline & HEPC74 & $1-69$ & 92 & $V_{\kappa} 1-5$ & 96 \\
\hline Chronically infected & $A R 3 C^{B}$ & $1-69$ & 86 & $V_{\kappa} 3-15$ & 95 \\
\hline
\end{tabular}

amino acid, without altering the other 12 somatic mutations in the sequence. We expressed the mAbs and measured binding to the panel of heterologous E1E2 variants proteins (Figure 5B). We also measured the effect of simultaneous reversion of all somatic mutations in the sequence encoding HCDR1, HCDR2, or HCDR3. Reversion of all mutations in HCDR1 or HCDR3 significantly reduced binding across the E1E2 panel, suggesting that these somatic mutations are important for binding to most heterologous E1E2 variants. Reversion of all somatic mutations in HCDR2 reduced binding to a subset of E1E2 variants. On evaluation of individual somatic mutations, reversion of glutamic acid 38 to alanine in HCDR1 significantly decreased binding of HEPC3 across the genotype 1 E1E2 panel. Similarly, reversion of threonine 65 to alanine in HCDR2 also significantly reduced binding across the panel, as did reversion of arginine 112 to serine in HCDR3. Interestingly, reversion of other individual somatic mutations had no detectable effect on binding to some E1E2 variants but profoundly reduced binding to others. For example, as shown in Figure $6 \mathrm{~A}$, reversion of leucine 30 to phenylalanine in HCDR1 or reinsertion of the germline-encoded glycine at the site of a deletion in HCDR2 (Del63G) had no effect on antibody binding to genotype 1a variants 1a09 or $1 \mathrm{a} 157$, but these reversions profoundly reduced binding to $1 \mathrm{~b}$ variants $1 \mathrm{~b} 09$ and $1 \mathrm{~b} 52$. Overall, reversion of each somatic mutation reduced binding to one or more variants in the heterologous panel, and each single amino acid reversion, except framework mutation $\mathrm{T} 87$ to alanine, reduced median binding across the E1E2 panel relative to mature HEPC3. These results, together with the almost complete loss of binding of HEPC3 H-RUA to all E1E2 variants, suggest that most or all somatic mutations present in the heavy chain of HEPC 3 contribute in combination to the breadth of 22 recognition by the bNAb.

We also performed quantitative kinetic binding analysis with the panel of HEPC 3 mAb variants and purified soluble J6 (genotype 2a) E2 protein (sE2) (Figure 6B and Supplemental Figure 6). Individual reversion of 11 of 13 heavy chain somatic mutations slightly reduced HEPC3 binding affinity for E2, as did reversion of all mutations simultaneously in HCDR1, HCDR2, or HCDR3. Interestingly, we observed large reductions in binding affinity with reversion of E38 to alanine and T66 to asparagine, two reversions that also consistently reduced binding across the heterologous genotype 1 panel. Notably, we also observed a reduction in binding affinity after reversion of L30 to F in HCDR1 and De163 to G in HCDR2, which supports the qualitatively similar effects of these reversions on antibody binding to genotype $1 \mathrm{~b}$ variants $1 \mathrm{~b} 09$ and $1 \mathrm{~b} 52$ (Figure 6A). Together, these data confirm that the majority of the heavy chain somatic mutations in HEPC3 are critical in combination for binding to heterologous E1E2 variants and that L30 and Del63 somatic mutations are particularly important for binding to subtype $1 \mathrm{~b}$ and $2 \mathrm{a}$ strains that are highly divergent from the genotype 1a virus infecting the HEPC3 donor.

Longitudinal evolution of autologous virus. To define the autologous E1E2 antigenic variants that favored selection and maturation of HEPC3 and investigate molecular mechanisms of viral clearance, we performed 
A
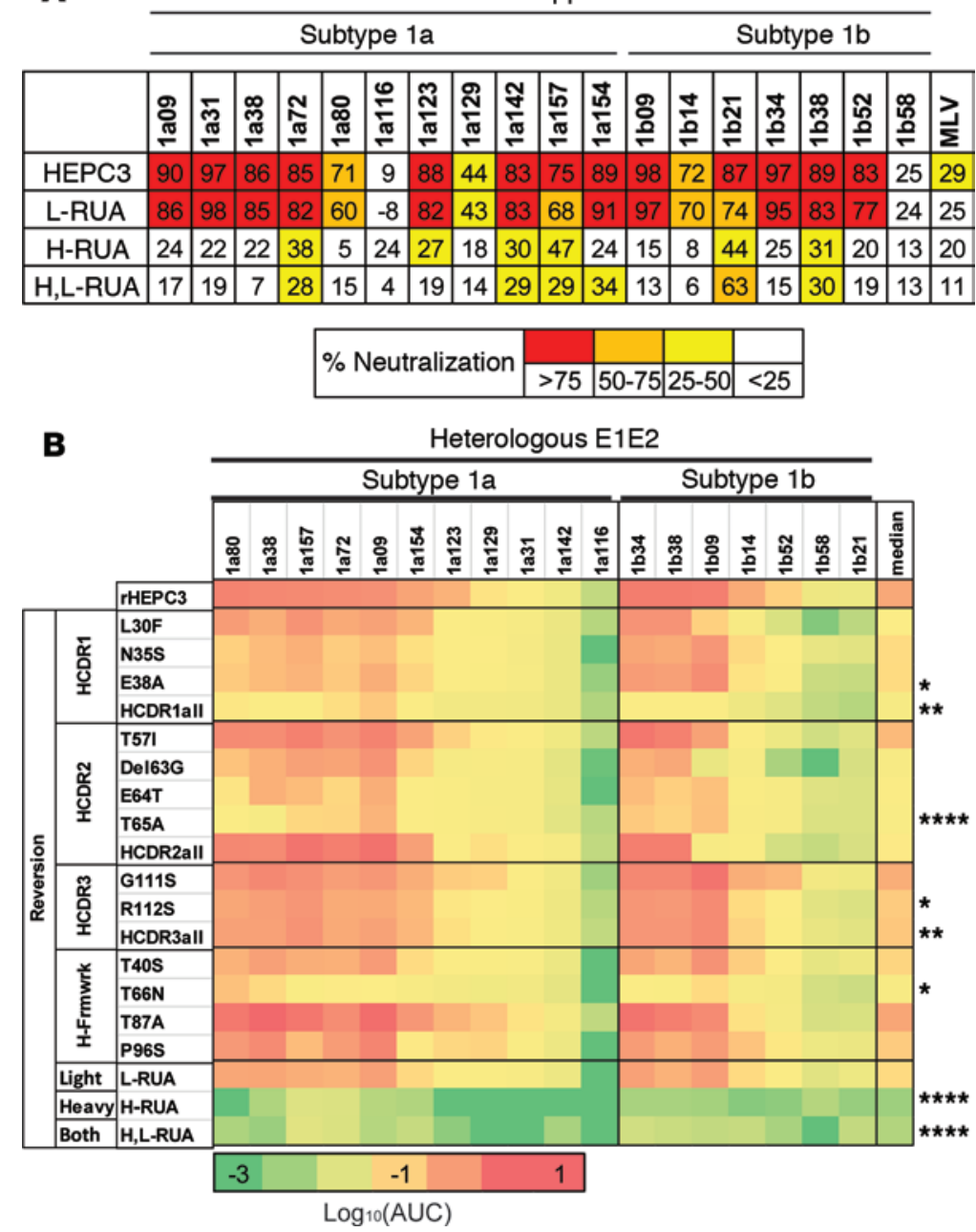

Figure 5. Role of somatic mutations in neutralization and binding of heterologous E1E2 proteins. (A) Neutralization of a heterologous genotype $1 \mathrm{HCV}$ pseudoparticle (HCVpp) panel by HEPC3, HEPC3 with reversion of all somatic mutations in the light chain variable region to the germline encoded amino acids (L-RUA), HEPC3 with reversion of all somatic mutations in the heavy chain variable region (H-RUA), or HEPC3 with reversion of all somatic mutations in both light and heavy chain variable regions (H,L-RUA) at a concentration of $50 \mu \mathrm{g} /$ $\mathrm{ml} \mathrm{mAb}$, measured in duplicate. (B) Binding of serial dilutions of the indicated mAbs to genotype 1E1E2 proteins, measured by ELISA. The individual reversion or combination of reversions introduced into HEPC3 is indicated on the vertical axis. The heatmap was generated using $\log _{10}$ (area under the curve) of binding of each mAb/E1E2 dilution series, which was measured in duplicate. Asterisks indicate significant differences between binding of each $\mathrm{mAb}$ to all E1E2 variants relative to binding of HEPC3 to the same E1E2 variants, measured by 1-way ANOVA with adjustment for multiple comparisons $\left({ }^{*} P<0.05,{ }^{* *} P<0.005,{ }^{* * *} P<0.0001\right)$.

extensive longitudinal sequencing of the HCV quasispecies of viruses present in plasma samples collected over time from subject 117. Plasma was isolated at 7 longitudinal time points - the first when HCV viremia initially was detected approximately 17 days after infection, and the last immediately prior to HCV clearance. Plasma RNA was isolated and RT-PCR was performed with single-genome amplification of 16-41 E1E2-spanning amp1icons per time point in order to define the viral diversity at each time point and the viral evolution over time. As shown in Figure 7, the subject was infected initially by at least 3 different $\mathrm{T} / \mathrm{F}$ viruses that differed from each other by $0.42 \%-1.23 \%$ in E1E2 nucleotide sequence and $0.72 \%-1.99 \%$ in E1E2 amino acid sequence. This esti-

mate of $3 \mathrm{~T} / \mathrm{F}$ genomes responsible for productive clinical infection is a minimum estimate, whose accuracy and precision are based on the numbers of sequences determined at the earliest sampling time points. In this subject, we determined 65 sequences in the initial 46 days of infection; previously described statistical power calculations (35) indicate that this provides a $>95 \%$ probability of detecting minor variant sequences present at a frequency of at least 5\%. Two of these viral lineages, T/F 1 and T/F 2, persisted and diversified over the next two sampling time points before they were apparently extinguished. The T/F 3 lineage persisted and evolved throughout the course of infection but exhibited a series of stringent population bottlenecks, such that only a single sublineage of day 194 sequences, exemplified by clone D194-FD13, gave rise to the last detectable virus population at day 388. Remarkably, day 388 sequences were comprised of a homogeneous expansion from a single genome present at day 285 , again indicative of a stringent population bottleneck. This lineage, D388-5A12, was last detected at the final sampling time point when HCV viral load had already declined to $754 \mathrm{IU} / \mathrm{ml}$ just prior to its extinction. The ratio of nonsynonymous to synonymous changes was high in genes encoding E1E2, suggesting positive selection (Supplemental Figure 7). The majority of nonsynonymous changes occurred in the hypervariable region 1 (HVR1) of E2. Outside of HVR1, 6 amino acid changes in E2 became fixed in the viral quasispecies over time (Supplemental Figure 8). Notably, 4 of these amino acid changes fell in a region of E2 spanned by the HEPC3-binding epitope, indicating selective pressure at this locus. E1E2 variants representative of all viral clades observed throughout the course of infection were cloned and expressed for binding studies.

Somatic mutations and recognition of autologous E1E2. We measured binding with an ELISA of mature HEPC3, HEPC3 H-RUA, and HEPC3 H,L-RUA to each of the 21 longitudinal autologous E1E2 variants (Figure 8). Remarkably, mature HEPC3 showed binding above background to all autologous variants, including the variants circulating immediately prior to viral clearance, suggesting that this $\mathrm{mAb}$ may have 
A
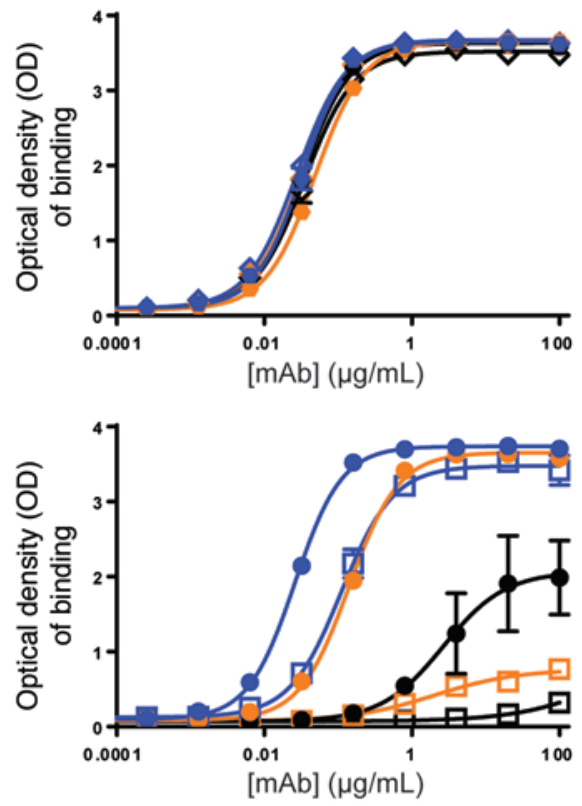

B

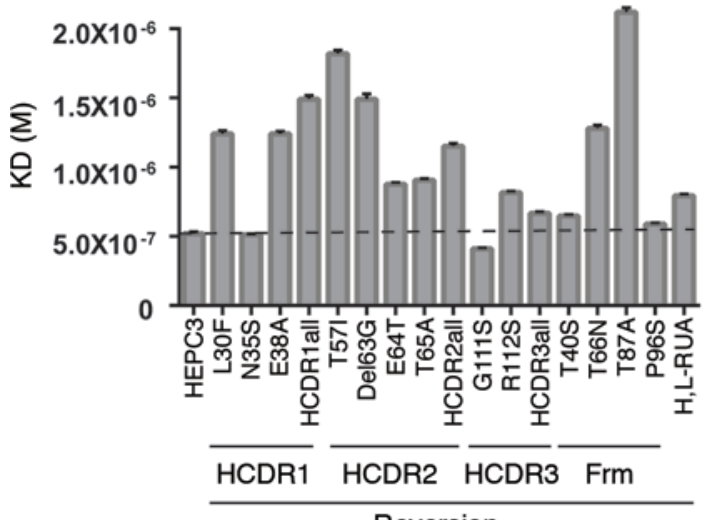

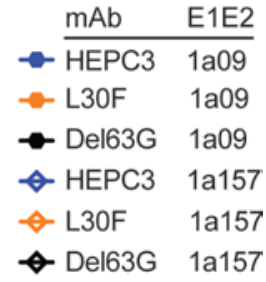

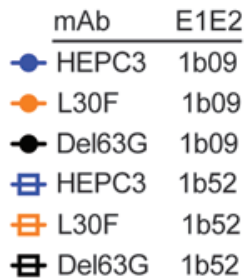

Figure 6. HCV strain-specific effects of bNAb somatic mutations. (A) Binding of serial dilutions of HEPC3 or the indicated HEPC3 mAb variants to 4 different genotype 1 E1E2 protein variants, measured by ELISA. Values are means of duplicate wells, and error bars indicate standard deviations. (B) Kinetic binding analysis of HEPC3 and HEPC3 mAb variants and soluble J6 strain (genotype 2a) E2 protein. Dissociation constants (KD) for each mAb are shown. Error bars represent the standard error of the mean, which was calculated using a global fit mode that includes several analyte concentrations. Single amino acid reversions in HEPC3 are grouped by their location in HCDR1, HCDR2, HCDR3, or framework regions (Frm).

contributed to clearance of infection. HEPC 3 H,L-RUA, with all somatic mutations in both heavy and light chain reverted to the germline-encoded sequence, lost detectable binding to 18 of 21 autologous variants but retained binding to 2 of $3 \mathrm{~T} / \mathrm{F}$ E1E2 variants and to a third variant also present at day 17 after infection. HEPC3 H-RUA showed a very similar pattern of binding to HEPC3 H,L-RUA, consistent with testing against heterologous E1E2 showing that somatic mutations in the heavy chain are more important than light chain somatic mutations for the neutralizing breadth of this $b N A b$. Taken together, these data suggest that the HEPC3 lineage may have arisen through binding of the unmutated ancestor of HEPC3 to T/F virus present very early after infection and that B cell clones with somatic mutations necessary for neutralizing breadth were likely selected by more resistant E1E2 variants circulating later in infection.

\section{Discussion}

These studies suggest that early emergence in diverse individuals of commonly occurring genetically related B cell clones encoding HCV-specific bNAbs with sparse somatic mutations is associated with spontaneous viral clearance. The data suggest a rational epitope-based vaccine approach may be highly feasible for HCV. To study the immune mechanism associated with clearance, we isolated and characterized bNAbs from subjects who spontaneously cleared HCV infection. We found that the intragenotypic and cross-genotypic neutralizing breadth of these mAbs compares favorably with the most potent anti-HCV bNAbs described to date. We also found that the binding epitopes of $3 \mathrm{mAbs}$ isolated from two different subjects were remarkably similar to each other and to the binding epitope of the well-characterized bNAb AR3C (from a third subject), suggesting that the $\mathrm{V}_{\mathrm{H}}$ 1-69 antibody gene segment commonly encodes a public $\mathrm{B}$ cell clonotype that favors early recogni- 


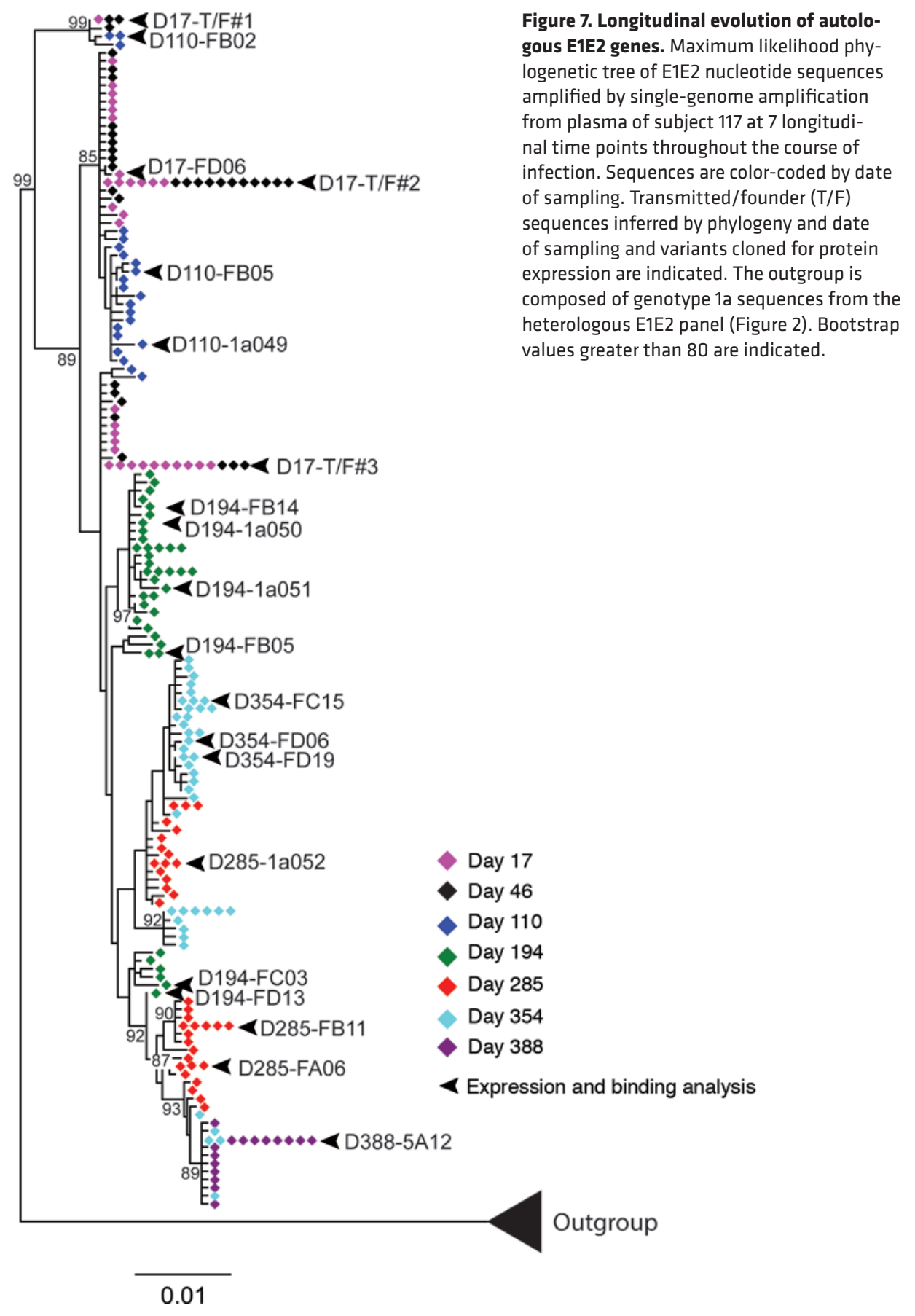

tion of this AR3C/HEPC3 epitope. Remarkably, the antibody genes encoding these bNAbs were not extensively somatically mutated, but the few somatic mutations present were critical in combination for facilitating the neutralizing breadth of one of the most potent bNAbs, HEPC3, as well as recognition of autologous viral variants circulating prior to viral clearance. Finally, we identified early T/F autologous envelopes that likely were responsible for selection of B cells expressing the HEPC3 unmutated ancestor antibody.

Multiple studies have now confirmed that several anti-HCV bNAbs are encoded by the $\mathrm{V}_{\mathrm{H}} 1-69$ antibody gene segment $(12,34)$. This study shows for the first time to our knowledge that $\mathrm{V}_{\mathrm{H}} 1-69$ commonly encodes a public $\mathrm{B}$ cell clonotype that can lead to $\mathrm{bNAb}$ development after a relatively brief period of infection, producing an antibody with recognition of an entire autologous viral quasispecies, thus providing what we believe to be the first potential mechanistic link between this B cell clonotype and spontaneous clearance of $\mathrm{HCV}$. These data suggest that antigens selecting B cells expressing $\mathrm{V}_{\mathrm{H}} 1$-69-encoded antibodies may be highly desirable for vaccine development. 

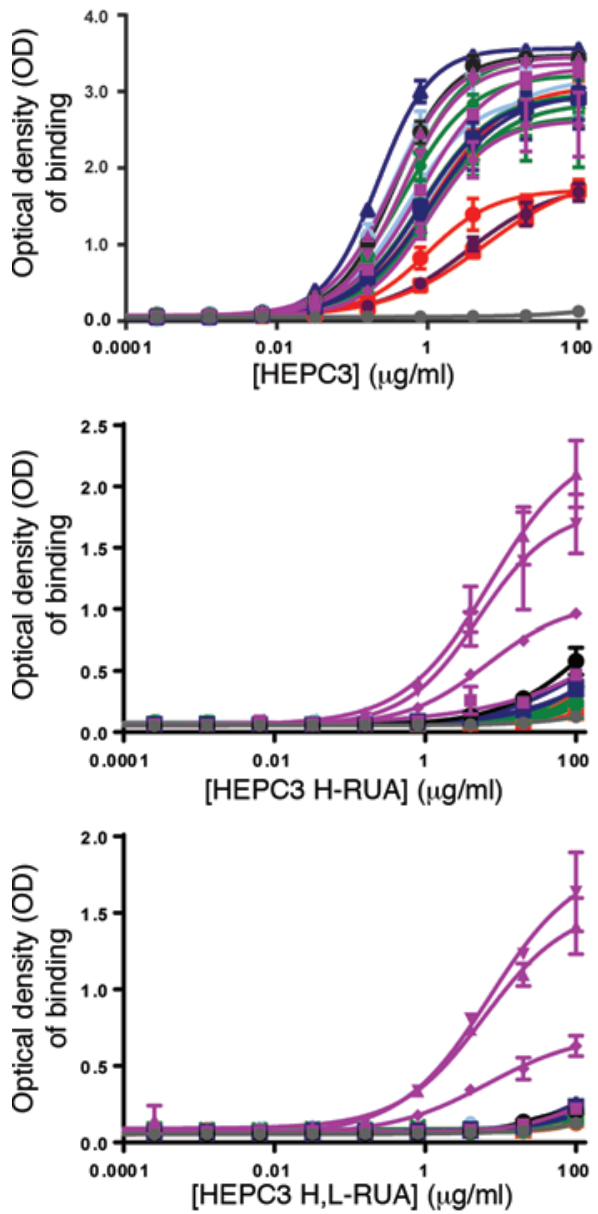

Autologous E1E2

(day of isolation-clone)

- D17-T/F\#1

— D17-T/F\#2

$\rightarrow \quad \mathrm{D} 17-\mathrm{T} / \mathrm{F} \# 3$

$\multimap$ D17-FD06

- D46-FA03

- D110-FB05

- D110-FB02

$\rightarrow \quad$ D110-1a49

$\neg \quad$ D194-FB14

* D194-FB05

× D194-FC03

+ D194-FD13

* D194-1a50

- D194-1a51

D285-FA06

- D285-FB11

$\multimap \quad$ D285-1a52

D354-FC15

D354-FD19

D354-FD06

D388-5A12

isotype control

$\mathrm{mAb}$
Figure 8. Role of somatic mutations in binding of autologous E1E2 proteins. Binding of serial dilutions of HEPC3, HEPC3 with all heavy chain somatic mutations reverted to the germline-encoded amino acid (HEPC3 H-RUA), or HEPC3 with all somatic mutations reverted to the germline-encoded amino acid (HEPC3 H,L-RUA) to 21 unique autologous E1E2 proteins. Proteins are color-coded by date of sampling. Values are the means of duplicate wells, and error bars indicate standard deviations. Median binding of an isotype control antibody to all E1E2 variants is shown as a control for nonspecific binding.

It is noteworthy that these bNAbs achieved broad neutralizing activity without extensive somatic mutation and after only a year of persistent viral infection. This finding stands in contrast to the typical evolution of bNAbs against HIV, which tend to require extensive somatic mutation that develops after years of chronic infection $(21,22)$. These results may suggest that, despite the enormous genetic variation of HCV, some E1E2 epitopes are relatively conserved and accessible to antibody binding. The degree of somatic mutation necessary to generate HEPC3, HEPC43, or HEPC74 (which exhibit 92\%-95\% identity to inferred germline genes) is generally achievable with traditional vaccination strategies $(36,37)$, whereas the more extensive level of somatic mutation found in anti-HIV bNAbs or even the anti-HCV mAb AR3C is less likely to be achievable.

Elegant studies of longitudinal viral evolution have been performed in HIV-infected subjects who developed bNAbs, with the goal of informing sequential vaccination strategies $(21,22)$. The results of these studies have been sobering, as they showed extensive and complex viral evolution driving the late development of bNAbs against HIV. This study suggests that the antigens required for stimulation of bNAbs against HCV may be less complex. Excluding HVR1, which is not the target of the bNAbs isolated in this study, HCV E1E2 of the most divergent E1E2 variant isolated from subject 117 differed by only 7 of 546 amino acids ( 1 percent) from the $\mathrm{T} / \mathrm{F}$ viruses that initially infected subject 117 . It is remarkable that the epitopes presented by the initial $\mathrm{T} / \mathrm{F}$ strains along with these few mutations in E1E2 were adequate to facilitate selection of both the bNAb unmutated ancestor and the mature antibody, which is capable of binding and neutralizing HCV variants from multiple genotypes, which differ at approximately $30 \%$ of their amino acids.

In addition, this study suggests that subjects who spontaneously clear HCV infection have mAbs that are similar in potency, neutralizing breadth, and epitope recognition to $\mathrm{mAbs}$ developed later in infection by some subjects with persistent HCV infection. However, these mAbs developed early in those who cleared infection without requiring extensive somatic hypermutation, which may have allowed $\mathrm{mAbs}$ to contribute to clearance before viral diversity and antibody resistance became too extensive. Therefore, the timing of emergence of bNAbs may be a critical factor in their ability to contain infection and restrict the emergence of addi- 
tional viral diversity. The concentration of HEPC3-like mAbs present in plasma of these subjects at the time of clearance is not known, so further studies are needed to confirm a direct contribution of these mAbs to clearance of infection. However, phylogenetic bottlenecks in the virus of subject 117, with selection of mutations in E1E2 conferring partial HEPC3 resistance, provide intriguing evidence that HEPC3-like mAbs were present at sufficient concentrations during infection to exert physiologically relevant pressure on the virus. We observed some recognition of all autologous variants by HEPC3, but the other distinct bNAbs isolated from this donor likely also contributed to clearance. The mechanisms by which these bNAbs may contribute in combination to clearance of $\mathrm{HCV}$ infection warrant further investigation.

In summary, we have isolated bNAbs from subjects with broadly neutralizing serum who spontaneously cleared HCV infection. Multiple bNAbs bind to the same epitope and use the same heavy chain V gene allele, identifying a public B cell clonotype that favors early binding to a conserved neutralizing epitope. Remarkably, these bNAbs were encoded by antibody genes with few somatic mutations. These somatic mutations were critical for antibody neutralizing breadth and binding to autologous envelope variants circulating late in infection, but they were not required for binding of the HEPC3 unmutated ancestor to envelope proteins of early autologous T/F viruses. This study shows that anti-HCV bNAbs can achieve substantial breadth with relatively few somatic mutations, and it identifies $\mathrm{HCV}$ envelope variants that were sufficient for selection and maturation of an anti-HCV $b N A b$ in vivo. These data provide a roadmap to guide development of a vaccine capable of stimulating anti-HCV bNAbs with a physiologic number of somatic mutations characteristic of vaccine responses. The work also provides one of the first views of the molecular basis for antibody-mediated clearance of an antigenically diverse and evolving chronic viral infection in humans.

\section{Methods}

Study subjects. Plasma samples and PBMCs were obtained from subjects in the BBAASH cohort (38).

Source of reference bNAbs. HC84.26 (13) and HC33.8 (8) were gifts from Steven Foung (Stanford University School of Medicine, Stanford, California, USA). AR1A, AR2A, AR3A, AR3C (17), AR4A, and AR5A (9) were gifts from Mansun Law (The Scripps Research Institute, La Jolla, California, USA).

$H C V$ viral load and serology testing. HCV viral loads (IU/ml) were quantified after RNA extraction with the use of commercial real-time reagents (Abbot HCV Real-time Assay) migrated onto a research-based real-time PCR platform (Roche 480 Lightcycler). HCV seropositivity was determined using the Ortho HCV version 3.0 ELISA Test System (Ortho Clinical Diagnostics).

Generation of human hybridomas secreting $m A b s$. Human hybridomas were generated as described previously (39). In brief, cryopreserved PBMC samples from subjects 110 and 117 were transformed with EBV, CpG, and additional supplements. After 7 days, cells from each well of the 384-well culture plates were expanded into four 96-well culture plates using cell culture medium containing irradiated heterologous human PBMCs and incubated for an additional 4 days. B cell culture supernatants were screened in ELISAs with E1E2 cell lysates and in neutralization assays against autologous or heterologous HCV variants. Screening was performed against E1E2 variants 1a53 (autologous) and 1a154 (H77) (heterologous) for subject 117 and 1a53 (heterologous) and 1a38 (autologous) variants for subject 110. Cells from wells with supernatants reacting with HCV antigens were fused with HMMA2.5 myeloma cells using an electrofusion technique (40). After fusion, hybridoma cell lines were cloned by limited dilutions and single-cell fluorescence-activated cell sorting and expanded in post-fusion medium as previously described (39). HiTrap Protein G or HiTrap MabSelectSure columns were used to purify HCV-specific antibodies from filtered cell culture supernatants.

HCVpp production and neutralization assays. The panel of 19 heterologous genotype $1 \mathrm{HCVpp}$ has been described previously $(28,30)$. The HCVpp panels used to measure neutralizing breadth of plasma samples and mAbs were identical, except that two E1E2 clones used to screen plasma, $1 \mathrm{~b} 20$ and 1a114, were replaced in $\mathrm{mAb}$ experiments by related clones $1 \mathrm{~b} 21$ and $1 \mathrm{a} 116$, which gave more consistent HCVpp infectivity results. HCVpp were produced by lipofectamine-mediated transfection of HCV E1E2 and pNL4-3.Luc.R-E- plasmids into HEK293T cells as previously described (41, 42). Neutralization assays were performed as described previously (43). HCVpp were incubated for 1 hour with plasma at a 1:100 dilution or $\mathrm{mAb}$ at a final concentration of 50 or $10 \mu \mathrm{g} / \mathrm{ml}$ and then added in duplicate to Hep3B target cells for 5 to 6 hours before medium was changed. Infection was determined after 3 days by measurement of luciferase activity of cell lysates in RLU. The majority of HCVpp used in neutralization assays produced $>2 \times 10^{6} \mathrm{RLU}$, or more than 100 -fold above background produced by mock pseudoparticles lacking any E1E2. All HCVpp used in neutralization assays produced at least 200,000 RLU, which is at least 
10-fold above background. Percentage of neutralization was calculated as $\left(1-\mathrm{RLU}_{\text {test mAb }} / \mathrm{RLU}_{\text {control IgG }}\right)$ $\times 100$. Neutralization of MLV-pseudotyped particles was measured as a negative control.

HCV cc neutralization assays. Chimeric genotype 1-6 HCVcc constructs $(44,45)$ were gifts from Jens Bukh (Copenhagen University Hospital, Copenhagen, Denmark). HCVcc neutralization assays were performed as described elsewhere (46). Briefly, human hepatoma Huh7.5.1 cells (a gift from Jake Liang, NIH, Bethesda, Maryland, USA) were maintained in DMEM supplemented with $10 \%$ fetal bovine serum, $1 \%$ sodium pyruvate, and 1\% L-glutamate. 10,000 Huh7.5.1 cells per well were plated in flat-bottom 96-well tissue culture plates and incubated overnight at $37^{\circ} \mathrm{C}$. The following day, HCVcc were mixed with mAb (2.5fold dilutions started at $50 \mu \mathrm{g} / \mathrm{ml}$ ) and then incubated at $37^{\circ} \mathrm{C}$ for 1 hour. Medium was removed from the cells and replaced with $50 \mu 1 \mathrm{HCVcc} /$ antibody mixture. The plates were placed in a $\mathrm{CO}_{2}$ incubator at $37^{\circ} \mathrm{C}$ overnight, after which the HCVcc were removed and replaced with $100 \mu 1$ Huh7.5.1 medium and incubated for 48 hours at $37^{\circ} \mathrm{C}$. After 48 hours, medium was removed and cells were fixed and stained. Images were acquired and spot-forming units (SFU) were counted in the presence of $\mathrm{mAb}\left(\mathrm{HCV}_{\mathrm{cc}} \mathrm{SFU}_{\text {test }}\right)$ or nonspecific IgG $\left(\mathrm{HCV}_{\mathrm{cc}} \mathrm{SFU}_{\text {control }}\right)$ using an AID iSpot Reader Spectrum operating AID ELISpot Reader version 7.0. Percentage of neutralization was calculated as $100 \times\left[1-\left(\mathrm{HCV}_{\mathrm{cc}} \mathrm{SFU}_{\text {test }} / \mathrm{HCV}_{\mathrm{cc}} \mathrm{SFU}_{\text {control }}\right)\right]$.

HCV NS5A immunostaining. HCV NS5A immunostaining was conducted as described elsewhere (46). Briefly, cells were fixed with 4\% formaldehyde and then stained for HCV NS5A using primary anti-NS5A antibody 9E10 (a gift from Charles Rice, The Rockefeller University, New York City, New York, USA) at a 1:10,000 dilution for 1 hour at room temperature. Cells were washed twice with PBS and stained using secondary antibody Alexa Daylight 488-conjugated goat anti-mouse IgG (Life Technologies) at a 1:500 dilution for 1 hour at room temperature. Cells were washed twice in PBS and then stored covered in $100 \mu \mathrm{PBS}$ at $4^{\circ} \mathrm{C}$.

Shotgun mutagenesis epitope mapping. Comprehensive alanine-scanning mutagenesis of an expression construct for HCV E1/E2 (genotype 1a, strain H77) changed each residue to alanine (with alanine residues changed to serine) to create a library of clones, each representing an individual point mutant, covering 552 of 555 target E1/E2 residues. Each mutation was confirmed by DNA sequencing, and clones were arrayed into 384-well plates, one mutant per well, transfected into HEK-293T cells, and allowed to express for 22 hours.

Cells were fixed in $4 \%$ paraformaldehyde, permeabilized with $0.1 \%$ (wt/vol) saponin (Sigma-Aldrich) in PBS plus calcium and magnesium, and stained with mAbs diluted in 10\% normal goat serum (NGS; Sigma-A1drich) and $0.1 \% \mathrm{w} / \mathrm{v}$ saponin, $\mathrm{pH}$ 9.0. Primary $\mathrm{mAb}$ concentrations were determined using an independent immunofluorescence titration curve against wild-type HCV E1/E2 to ensure that signals were within the linear range of detection. The cells were incubated with anti-HCV antibody for 1 hour at $20^{\circ} \mathrm{C}$, followed by a $30-\mathrm{min}$ ute incubation with Alexa Fluor 488-conjugated secondary antibody (Jackson ImmunoResearch Laboratories) in $10 \%$ NGS. Cells were washed twice with PBS without calcium or magnesium and resuspended in Cellstripper (Cellgro) plus $0.1 \%$ BSA (Sigma-Aldrich). Cellular fluorescence was detected using the Intellicyt high-throughput flow cytometer (Intellicyt). Background fluorescence was determined by fluorescence measurement of vector-transfected control cells. mAb reactivities against each mutant $\mathrm{HCV}$ E1/E2 clone were calculated relative to wild-type E1/E2 reactivity by subtracting the signal from mock-transfected controls and normalizing to the signal from wild-type HCV E1/E2-transfected controls.

Mutated residues within critical clones were identified as critical to the mAb epitope if they did not support reactivity of the test $\mathrm{mAb}$ but did support reactivity of other control anti-HCV mAbs. This counter-screen strategy facilitates the exclusion of E1/E2 mutants that are locally misfolded or that have an expression defect $(47,48)$.

Hierarchical clustering of $m A$ bs based on correlations between neutralization profiles. Neutralization of each of $19 \mathrm{HCVpp}$ by each $\mathrm{mAb}$ was compared pairwise for all mAbs using Spearman correlation. Spearman rho and $P$ values then were used as input for hierarchical clustering as implemented in the "pvclust" package for R (http://cran.r-project.org/web/packages/pvclust/index.html) (49). Approximately unbiased values of greater than 95 are considered strongly supported by the data. This clustering, depicted as a tree, was also used to order a matrix of correlation values produced using the "corrplot" package for R (http:// cran.r-project.org/web/packages/corrplot/index.html) (50).

HCV E1E2 ELISA. mAb binding to E1E2 was quantitated using an ELISA as previously described (15). Briefly, 293T cells were transfected with E1E2 expression constructs. Cell lysates were harvested at 48 hours. Plates were coated with $500 \mathrm{ng}$ Galanthus nivalis lectin (Sigma-Aldrich) and blocked with PBS containing $0.5 \%$ Tween-20, $1 \%$ nonfat dry milk, and $1 \%$ goat serum, and E1E2-containing cell lysates were added. mAbs were assayed in duplicate at 5 -fold serial dilutions, starting at $100 \mu \mathrm{g} / \mathrm{ml}$, 
and binding was detected with HRP-conjugated anti-human IgG secondary antibody (BD Pharmingen 555788). ELISA used for hybridoma screening followed the same protocol with the following modifications: 384-well ELISA plates were coated with Galanthus nivalis lectin (Sigma-Aldrich) at $5 \mu \mathrm{g} /$ $\mathrm{ml}$ in Dulbecco's PBS (DPBS). Plates were blocked with a blocking solution consisted of $10 \mathrm{~g}$ powdered milk, $10 \mathrm{ml}$ goat serum, $100 \mathrm{ml} 10 \times$ DPBS, and $0.5 \mathrm{ml}$ Tween-20 mixed to a 1-1 final volume with distilled water. Autologous and heterologous E1E2 cell lysates were diluted in DPBS with $0.05 \%$ Tween-20 and added to plates. B cell culture supernatants from tissue-culture plates were transferred to ELISA plates coated with E1E2 lysates. For binding competitions with ELISA, E1E2 protein-coated ELISA wells were preincubated with $20 \mu \mathrm{g} / \mathrm{ml}$ of blocking bNAbs, followed by biotinylated mAbs at $2 \mu \mathrm{g} / \mathrm{ml}$, with binding of the biotinylated $\mathrm{bNAb}$ detected using streptavidin-horseradish peroxidase. The ratio of binding of each biotinylated $\mathrm{bNAb}$ in the presence of blocking $\mathrm{bNAb}$ to binding in the absence of blocking bNAb was calculated. For ELISA with denatured E1E2 protein, E1E2 was boiled for 5 minutes in Tris-buffered saline-10\% FCS containing 1.0\% sodium dodecyl sulfate and $50 \mathrm{mM}$ dithiothreitol, prior to addition to GNA-lectin-coated plates.

Sequence analysis of antibody variable region genes. Total cellular RNA was extracted from clonal hybridomas that produced HCV antibodies, and RT-PCR reaction was performed using mixtures of primers designed to amplify all heavy chain or light chain antibody variable regions. Antibody variable gene sequence analysis was performed as previously described (39). Heavy and light chain antibody variable region sequences were analyzed using the IMGT/V-Quest program $(51,52)$.

HEPC3 mutagenesis and antibody expression. The genes encoding HEPC3 heavy or light chain variable regions were synthesized and cloned into a mammalian expression plasmid vector for full-length IgG1 by GenScript. Site-directed mutagenesis to revert individual somatic mutations was performed using the QuikChange Lightning Site-Directed Mutagenesis kit (Agilent). Transient expression of HEPC3 variants was done in Freestyle 293-F cells (Thermo Fisher Scientific). Briefly, equal amounts of heavy and light chain DNA were mixed with polyethylenimine (PEI; Polysciences Inc.) at 2:1 ratio of PEI to DNA and the DNAPEI complexes were added to Freestyle cells. $200 \mathrm{ml}$ or $300 \mathrm{ml}$ of culture was used for each variant, and supernatants were collected on day 6 after transfection. mAbs were harvested from the supernatant using HiTrap MabSelectSure columns (Life Technologies).

Biolayer interferometry-based $K_{D}$ measurements. HEPC3 variant binding affinities were determined using an Octet RED biosensor (ForteBio). HEPC3 variants were diluted in $1 \times$ kinetic buffer to $10 \mu \mathrm{g} / \mathrm{ml}$ and immobilized onto anti-human IgG Fc Capture sensors (ForteBio). The kinetic experiments included five steps: (a) baseline (60 seconds); (b) HEPC3 variants loading onto sensors (60 seconds); (c) second baseline (60 seconds); (d) association of J6 E2 (300 seconds); and (e) dissociation of J6 E2 (300 seconds). Fitting curves were constructed using ForteBio Data Analysis 7.0 software using a 1:1 binding model, and background subtraction was used for correction.

Single HCV genome amplification. HCV hemigenomes from plasma virus were amplified by RT-PCR after limiting dilution to ensure single-genome amplification, using previously described methods (53). PCR products were gel extracted and directly Sanger sequenced. E1E2 was PCR amplified from hemigenomic single-genome amplification amplicons of interest and cloned as previously described (28). All E1E2 clones were Sanger sequenced to confirm that errors had not been introduced by the additional PCR step. All original sequence data were deposited in GenBank (accession KY965445-KY965807). Two E1E2 sequences included in this study, GenBank accession FJ828970.1 and FJ828971.1, were previously described (43).

E1E2 sequence analysis. Nucleotide sequences spanning E1E2 were trimmed and aligned using MUSCLE, with the alignment manually adjusted in BioEdit. The phylogenetic tree was inferred from nucleotide sequences using the maximum likelihood method based on the Tamura 3-parameter model (54), gamma distributed. The tree with highest log likelihood is shown with branches drawn to scale. Initial tree(s) for the heuristic search were obtained automatically by applying neighbor-joining and BioNJ algorithms to a matrix of pairwise distances estimated using the maximum composite likelihood approach and then selecting the topology with superior log likelihood value. 500 bootstrap tests were performed. Analyses were implemented in the Mega6 program (http://www.megasoftware.net). Sliding window nonsynonymous/ synonymous analysis was performed by the Nei-Gojobori method implemented in VarPlot (http://sray. med.som.jhmi.edu), with 20-codon windows and 1-codon steps. T/F genomes were inferred as previously described $(53,55)$. Highlighter plots were generated using aligned E1E2 amino acid sequences and the Highlighter tool at the Los Alamos HIV database (http://www.lanl.gov/). 
Statistics. Neutralization curves for HCVcc and ELISA binding curves were fit by nonlinear regression in Graphpad Prism, and mAb 50\% inhibitory concentration (neutralization assay) or area under the curve (binding assay) was calculated based on that curve. $\log _{10}$ (area under the curve) of binding to each of the 19 genotype 1 heterologous E1E2 variants was compared between HEPC 3 and HEPC3 variant mAbs by paired 1 -way ANOVA analysis with correction for multiple comparisons. $P<0.05$ were considered significant.

Study approval. The protocol was approved by the Institutional Review Board of the Johns Hopkins Hospital, and informed consent was obtained from all study participants.

\section{Author contributions}

JRB designed the study, conducted experiments, analyzing data, and wrote the manuscript; AIF designed the study, conducted experiments, analyzed data, and wrote the manuscript; VJC conducted experiments and analyzed data; HL conducted experiments; LNW conducted experiments; AES conducted experiments; SW conducted experiments; GHL analyzed data; NK conducted experiments; LL conducted experiments; RL conducted experiments; ALC analyzed data and wrote the manuscript; JMP conducted experiments; BJD conducted experiments and analyzed data; GMS analyzed data and wrote the manuscript; SCR designed the study and wrote the manuscript; and JEC designed the study, analyzed data, and wrote the manuscript.

\section{Acknowledgments}

This project received support from the US NIH (grants K08 AI102761, U19 AI088791, and 1P30AI094189 and contracts HHSN272200900055C and HHSN272201400058C). The project was supported by National Center for Research Resources grant UL1 RR024975 and is now supported by the National Center for Advancing Translational Sciences (grant 2 UL1 TR000445). Flow cytometry experiments were performed at the Vanderbilt University Medical Center Flow Cytometry Shared Resource, supported by NIH grants P30 CA68485 and DK058404, and viral sequencing was performed at the University of Pennsylvania Center for AIDS Research Sequencing Core, supported by NIH grant P01 AI45008. The content is solely the responsibility of the authors and does not necessarily represent the official views of the NIH. We thank the Vanderbilt Clinical Trials Center for regulatory support. We thank Frances Smith-House for technical support. We thank Daved Fremont and Michael Diamond of Washington University at St. Louis for recombinant E2 protein used in biosensor assays.

Address correspondence to: James E. Crowe, Jr., Vanderbilt Vaccine Center, 11475 MRB IV, 2213 Garland Avenue, Nashville, Tennessee 37232-0417, USA. Phone: 615.343.8064; E-mail: james.crowe@vanderbilt.edu.

1. Mohd Hanafiah K, Groeger J, Flaxman AD, Wiersma ST. Global epidemiology of hepatitis C virus infection: new estimates of age-specific antibody to HCV seroprevalence. Hepatology. 2013;57(4):1333-1342.

2. Cox AL. MEDICINE. Global control of hepatitis C virus. Science. 2015;349(6250):790-791.

3. Zibbell JE, et al. Increases in hepatitis $C$ virus infection related to injection drug use among persons aged $\leq 30$ years - Kentucky, Tennessee, Virginia, and West Virginia, 2006-2012. MMWR Morb Mortal Wkly Rep. 2015;64(17):453-458.

4. Conrad C, et al. Community Outbreak of HIV Infection Linked to Injection Drug Use of Oxymorphone--Indiana, 2015. MMWR Morb Mortal Wkly Rep. 2015;64(16):443-444.

5. Suryaprasad AG, et al. Emerging epidemic of hepatitis $C$ virus infections among young nonurban persons who inject drugs in the United States, 2006-2012. Clin Infect Dis. 2014;59(10):1411-1419.

6. Hadlock KG, et al. Human monoclonal antibodies that inhibit binding of hepatitis C virus E2 protein to CD81 and recognize conserved conformational epitopes. J Virol. 2000;74(22):10407-10416.

7. Keck ZY, et al. A point mutation leading to hepatitis $\mathrm{C}$ virus escape from neutralization by a monoclonal antibody to a conserved conformational epitope. J Virol. 2008;82(12):6067-6072.

8. Keck Z, et al. Cooperativity in virus neutralization by human monoclonal antibodies to two adjacent regions located at the amino terminus of hepatitis C virus E2 glycoprotein. $J$ Virol. 2013;87(1):37-51.

9. Giang E, et al. Human broadly neutralizing antibodies to the envelope glycoprotein complex of hepatitis C virus. Proc Natl Acad Sci USA. 2012;109(16):6205-6210.

10. Krey T, Meola A, Keck ZY, Damier-Piolle L, Foung SK, Rey FA. Structural basis of HCV neutralization by human monoclonal antibodies resistant to viral neutralization escape. PLoS Pathog. 2013;9(5):e1003364.

11. Johansson DX, et al. Human combinatorial libraries yield rare antibodies that broadly neutralize hepatitis C virus. Proc Natl Acad Sci USA. 2007;104(41):16269-16274.

12. Merat SJ, et al. Hepatitis C virus broadly neutralizing monoclonal antibodies isolated 25 years after spontaneous clearance. PLoS ONE. 2016;11(10):e0165047.

13. Keck ZY, et al. Human monoclonal antibodies to a novel cluster of conformational epitopes on HCV E2 with resistance to neu- 
tralization escape in a genotype 2a isolate. PLoS Pathog. 2012;8(4):e1002653.

14. Keck ZY, et al. Mapping a region of hepatitis C virus E2 that is responsible for escape from neutralizing antibodies and a core CD81-binding region that does not tolerate neutralization escape mutations. J Virol. 2011;85(20):10451-10463.

15. Keck ZY, et al. Mutations in hepatitis C virus E2 located outside the CD81 binding sites lead to escape from broadly neutralizing antibodies but compromise virus infectivity. J Virol. 2009;83(12):6149-6160.

16. Kong L, et al. Structural basis of hepatitis C virus neutralization by broadly neutralizing antibody HCV1. Proc Natl Acad Sci USA. 2012;109(24):9499-9504.

17. Law M, et al. Broadly neutralizing antibodies protect against hepatitis C virus quasispecies challenge. Nat Med. 2008;14(1):25-27.

18. Morin TJ, et al. Human monoclonal antibody HCV1 effectively prevents and treats HCV infection in chimpanzees. PLoS Pathog. 2012;8(8):e1002895.

19. de Jong YP, et al. Broadly neutralizing antibodies abrogate established hepatitis C virus infection. Sci Transl Med. 2014;6(254):254ra129.

20. Burton DR, Mascola JR. Antibody responses to envelope glycoproteins in HIV-1 infection. Nat Immunol. 2015;16(6):571-576.

21. Bhiman JN, et al. Viral variants that initiate and drive maturation of V1V2-directed HIV-1 broadly neutralizing antibodies. Nat Med. 2015;21(11):1332-1336.

22. Bonsignori M, et al. Maturation Pathway from Germline to Broad HIV-1 Neutralizer of a CD4-Mimic Antibody. Cell. 2016;165(2):449-463.

23. Thomas DL, et al. Genetic variation in IL28B and spontaneous clearance of hepatitis C virus. Nature. 2009;461(7265):798-801.

24. Martell M, et al. Hepatitis C virus (HCV) circulates as a population of different but closely related genomes: quasispecies nature of HCV genome distribution. J Virol. 1992;66(5):3225-3229.

25. Simmonds $P$, et al. Classification of hepatitis $C$ virus into six major genotypes and a series of subtypes by phylogenetic analysis of the NS-5 region. J Gen Virol. 1993;74(Pt 11):2391-2399.

26. Pybus OG, Charleston MA, Gupta S, Rambaut A, Holmes EC, Harvey PH. The epidemic behavior of the hepatitis C virus. Science. 2001;292(5525):2323-2325.

27. Ray SC, Thomas DL. Hepatitis C. In: Mandell GL, Bennett JE, Dolin R eds. Mandell, Douglas, and Bennett's Principles and Prac tice of Infectious Diseases. Philadelphia: Chuchill Livingstone; 2010:2157-85.

28. Osburn WO, et al. Clearance of hepatitis $\mathrm{C}$ infection is associated with the early appearance of broad neutralizing antibody responses. Hepatology. 2014;59(6):2140-2151.

29. Pestka JM, et al. Rapid induction of virus-neutralizing antibodies and viral clearance in a single-source outbreak of hepatitis C. Proc Natl Acad Sci USA. 2007;104(14):6025-6030.

30. Bailey JR, et al. Naturally selected hepatitis C virus polymorphisms confer broad neutralizing antibody resistance. J Clin Invest. 2015;125(1):437-447.

31. Kong L, et al. Hepatitis C virus E2 envelope glycoprotein core structure. Science. 2013;342(6162):1090-1094.

32. Owsianka AM, et al. Identification of conserved residues in the E2 envelope glycoprotein of the hepatitis $\mathrm{C}$ virus that are critical for CD81 binding. J Virol. 2006;80(17):8695-8704.

33. Georgiev IS, et al. Delineating antibody recognition in polyclonal sera from patterns of HIV-1 isolate neutralization. Science. 2013;340(6133):751-756.

34. Chan CH, Hadlock KG, Foung SK, Levy S. V(H)1-69 gene is preferentially used by hepatitis C virus-associated B cell lymphomas and by normal B cells responding to the E2 viral antigen. Blood. 2001;97(4):1023-1026.

35. Keele BF, et al. Identification and characterization of transmitted and early founder virus envelopes in primary HIV-1 infection. Proc Natl Acad Sci USA. 2008;105(21):7552-7557.

36. Scherer EM, Smith RA, Simonich CA, Niyonzima N, Carter JJ, Galloway DA. Characteristics of memory B cells elicited by a highly efficacious HPV vaccine in subjects with no pre-existing immunity. PLoS Pathog. 2014;10(10):e1004461.

37. Wang C, et al. B-cell repertoire responses to varicella-zoster vaccination in human identical twins. Proc Natl Acad Sci USA. 2015;112(2):500-505.

38. Cox AL, et al. Prospective evaluation of community-acquired acute-phase hepatitis C virus infection. Clin Infect Dis. 2005;40(7):951-958.

39. Flyak AI, et al. Mechanism of human antibody-mediated neutralization of Marburg virus. Cell. 2015;160(5):893-903.

40. Yu X, McGraw PA, House FS, Crowe JE. An optimized electrofusion-based protocol for generating virus-specific human monoclonal antibodies. J Immunol Methods. 2008;336(2):142-151.

41. Hsu M, et al. Hepatitis C virus glycoproteins mediate pH-dependent cell entry of pseudotyped retroviral particles. Proc Natl Acad Sci USA. 2003;100(12):7271-7276.

42. Logvinoff C, et al. Neutralizing antibody response during acute and chronic hepatitis C virus infection. Proc Natl Acad Sci USA 2004;101(27):10149-10154.

43. Dowd KA, Netski DM, Wang XH, Cox AL, Ray SC. Selection pressure from neutralizing antibodies drives sequence evolution during acute infection with hepatitis C virus. Gastroenterology. 2009;136(7):2377-2386.

44. Gottwein JM, et al. Development and characterization of hepatitis C virus genotype 1-7 cell culture systems: role of CD81 and scavenger receptor class B type I and effect of antiviral drugs. Hepatology. 2009;49(2):364-377.

45. Scheel TK, et al. Development of JFH1-based cell culture systems for hepatitis C virus genotype 4a and evidence for cross-genotype neutralization. Proc Natl Acad Sci USA. 2008;105(3):997-1002.

46. Wasilewski LN, et al. A Hepatitis C virus envelope polymorphism confers resistance to neutralization by polyclonal sera and broadly neutralizing monoclonal antibodies. J Virol. 2016;90(7):3773-3782.

47. Paes C, et al. Atomic-level mapping of antibody epitopes on a GPCR. J Am Chem Soc. 2009;131(20):6952-6954.

48. Davidson E, Doranz BJ. A high-throughput shotgun mutagenesis approach to mapping B-cell antibody epitopes. Immunology. 2014;143(1):13-20.

49. Suzuki R, Shimodaira H. Pvclust: an R package for assessing the uncertainty in hierarchical clustering. Bioinformatics. 2006;22(12):1540-1542.

50. Friendly M. Corrgrams: Exploratory displays for correlation matrices. Am Stat. 2002;56:316-324. 
51. Brochet X, Lefranc MP, Giudicelli V. IMGT/V-QUEST: the highly customized and integrated system for IG and TR standardized V-J and V-D-J sequence analysis. Nucleic Acids Res. 2008;36(Web Server issue):W503-W508.

52. Giudicelli V, Brochet X, Lefranc MP. IMGT/V-QUEST: IMGT standardized analysis of the immunoglobulin (IG) and T cell receptor (TR) nucleotide sequences. Cold Spring Harb Protoc. 2011;2011(6):695-715.

53. Li H, et al. Elucidation of hepatitis $\mathrm{C}$ virus transmission and early diversification by single genome sequencing. PLoS Pathog. 2012;8(8):e1002880.

54. Tamura K, Kumar S. Evolutionary distance estimation under heterogeneous substitution pattern among lineages. Mol Biol Evol. 2002;19(10):1727-1736.

55. Li H, et al. Single-genome sequencing of hepatitis $\mathrm{C}$ virus in donor-recipient pairs distinguishes modes and models of virus transmission and early diversification. $J$ Virol. 2015;90(1):152-166. 